\title{
Supporting Information for Exploring the Solvation of Acetic Acid in Water Using Liquid Jet X - ray Photoelectron Spectroscopy and Core Level Electron Binding Energy Calculations
}

Jared P. Brucet, Kimberly Zhang, Sree Ganesh Balasubramani, Amanda R. Haines, Randima P. Galhenage, Vamsee K. Voora"t, Filipp Furche, and John C. Hemminger*

Department of Chemistry, University of California, Irvine, Irvine, California, USA 92697

Email:jchemmin@uci.edu

Current Addresses:

Department of Interface Science, Fritz Haber Institute, Berlin, Germany 14195

"t Department of Chemical Sciences, Tata Institute of Fundamental Research, Homi Bhabha Road, Colaba, Mumbai 400005
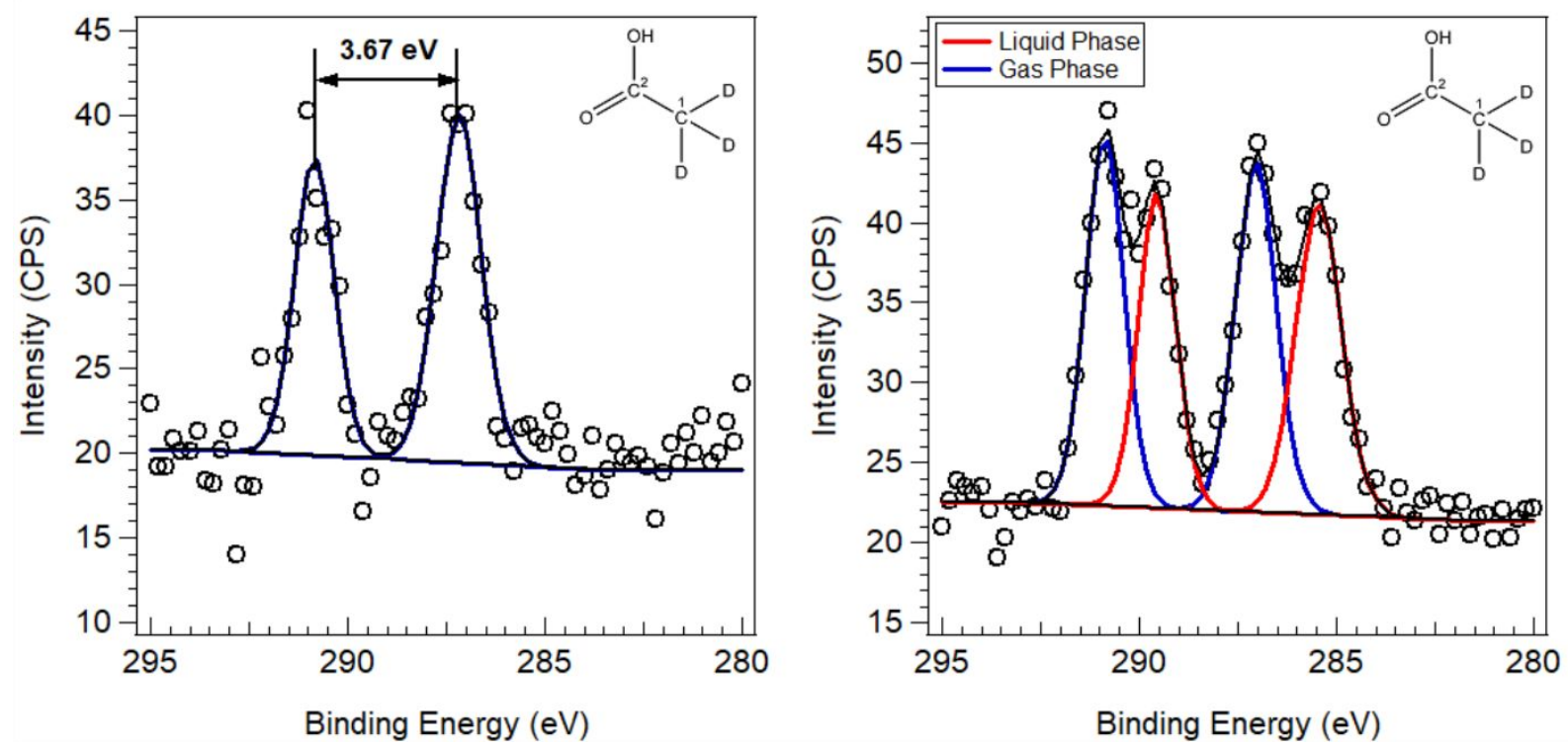

Figure 1: C 1s LJ-APXPS spectra of (a) gas phase $\mathrm{d}^{4}$-acetic acid above the liquid jet (b) $5 \mathrm{M} \mathrm{d} \mathrm{d}^{4}$-acetic acid in aqueous solution showing both the gas phase acetic acid and acetic acid dissolved in the solution. 
Table 1: Summary of $\mathrm{d}^{4}$-acetic acid fits of $\mathrm{C} 1 \mathrm{~s}$ data. Errors are found through Monte Carlo analysis and propagation of errors to find $\triangle \mathrm{BE}$.

\begin{tabular}{|c|c|c|c|c|}
\hline & $\begin{array}{c}\text { C1s carboxyl } \\
\text { (gas) }\end{array}$ & $\begin{array}{c}\text { C1s methyl } \\
\text { (gas) }\end{array}$ & $\begin{array}{c}\text { C1s carboxyl } \\
\text { (liquid) }\end{array}$ & $\begin{array}{c}\text { C1s methyl } \\
\text { (liquid) }\end{array}$ \\
\hline $\begin{array}{c}\text { Binding Energy } \\
\text { (eV) }\end{array}$ & $290.92 \pm 0.06$ & $287.25 \pm 0.05$ & $289.71 \pm 0.06$ & $285.60 \pm 0.05$ \\
\hline FWHM (eV) & $1.07 \pm 0.22$ & $1.17 \pm 0.19$ & $1.10 \pm 0.11$ & $1.35 \pm 0.14$ \\
\hline$\Delta$ BE (eV) & \multicolumn{2}{|r|}{$3.67 \pm 0.08$} & \multicolumn{2}{|c}{$4.11 \pm 0.08$} \\
\hline
\end{tabular}

Table 2: Addition of $\mathrm{d}^{4}$-acetic acid $\triangle \mathrm{BE}$ into Table 2 in the main manuscript. These values were found based on a fit of the liquid phase and gas phase liquid jet data

\begin{tabular}{|c|c|c|}
\hline Number of Solvent Molecules & Acetic acid $\triangle B E$ in Water & Acetate $\triangle \mathrm{BE}$ in Water \\
\hline Gas Phase & 3.58 & - \\
\hline 1 & $3.76 \pm 0.17$ & $3.03 \pm 0.12$ \\
\hline 2 & $3.82 \pm 0.16$ & $3.06 \pm 0.15$ \\
\hline 3 & $3.77 \pm 0.16$ & $3.13 \pm 0.11$ \\
\hline 4 & $3.82 \pm 0.18$ & $3.14 \pm 0.13$ \\
\hline 8 & - & 3.32 \\
\hline 10 & - & 3.40 \\
\hline 20 & - & 3.45 \\
\hline Experiment Liquid & $4.02 \pm 0.11$ & $3.49 \pm 0.06$ \\
\hline Experiment Gas & $3.90 \pm 0.11$ & - \\
\hline Experiment d $d^{4}$ Gas & $3.67 \pm 0.08$ & - \\
\hline Experiment $d^{4}$ Liquid & $4.11 \pm 0.08$ & - \\
\hline
\end{tabular}


Trajectory data and computational results are available free of charge from Zhang, Kimberly et al. (2021), Exploring the Solvation of Acetic Acid in Water Using Liquid Jet X-ray Photoelectron Spectroscopy and Core Level Electron Binding Energy Calculations, Dryad, Dataset, https://doi.org/10.7280/D1DQ21 